\title{
Towards an Axiomatization of the Core-Center*
}

\author{
Julio GonZÁlez-DíAZ \\ Kellogg School of Management (CMS-EMS), Northwestern University \\ and \\ Research Group in Economic Analysis, University of Vigo \\ Estela SÁnchez-RodRÍGUeZ \\ Department of Statistics and Operations Research, University of Vigo \\ Published in European Journal of Operational Research (2009) 195, 449-459 \\ Published version available at http://www.sciencedirect.com \\ DOI $10.1016 /$ j.ejor.2008.02.004
}

\begin{abstract}
The core-center is an allocation rule introduced in González-Díaz and SánchezRodríguez (2007) for the class of games with a nonempty core. In this paper we present a weighted additivity axiom, which we call trade-off property, and use it to obtain two characterizations of the core-center.
\end{abstract}

Keywords. COOPERATive TU Games, CORE, CORE-CEnter, AXIOMATization, Center OF GRAVITY

\section{Introduction}

In González-Díaz and Sánchez-Rodríguez (2007), the core-center, a new allocation rule for the class of games with nonempty core is introduced and a detailed analysis of its properties is carried out. In this paper we present two axiomatic characterizations of the core-center.

*Acknowledgments. The authors are indebted to José Carlos Díaz Ramos, Roi Docampo Álvarez and two anonymous referees for their suggestions. Support from the Spanish Ministry for Science and Education through projects SEJ2005-07637-C02-02 and from the Xunta de Galicia under project PGIDT03PXIC20701PN is gratefully acknowledged. The research of Julio González-Díaz has also been supported by a Marie Curie International Fellowship within the 6th European Community Framework Programme.

${ }^{\dagger}$ Corresponding author: Kellogg School of Management (CMS-EMS), 5100 Leverone Hall, 2001 Sheridan Road. Evanston, IL 60208-2014. United States. email: julio@northwestern.edu 
The key property for both characterizations is a weighted additivity that is based on a principle of fairness with respect to the core, which we call trade-off property.

In the first characterization we show that the trade-off property along with a natural symmetry property suffices to characterize the core-center for the class of three-player convex games. In the second result we characterize the core-center for the general class of $n$-player games with nonempty core. This second characterization of the core-center consists of four properties. Three of them are quite standard: efficiency, continuity and a weak symmetry property. The fourth property is strong trade-off, an strengthening of the trade-off property. The proof of this second result has a certain parallelism with the characterization of the Shapley value based on the additivity property (Shapley, 1953). First, we prove the result for games with a simplicial core, which play the role of the unanimity games in Shapley's characterization. Second, we prove the result for arbitrary games by means of simplicial dissections of their cores.

The structure of this paper is as follows. In Section 2 we introduce the preliminary game theoretical concepts along with the definition of the core-center. In Section 3 we define and discuss both the trade-off property and the strong trade-off property. In Section 4 we present the characterization of the core-center for three-player convex games. In Section 5 we present the characterization for general $n$-player games with nonempty core.

\section{Game Theory Background}

A transferable utility or TU game is a pair $(N, v)$, where $N:=\{1, \ldots, n\}$ is a set of players and $v: 2^{N} \rightarrow \mathbb{R}$ is a function assigning, to each coalition $S \subseteq N$, its worth $v(S)$. By convention, $v(\emptyset)=0$. Let $G^{n}$ denote the set of $n$-player TU games. Since each game assigns a real value to each nonempty subset of $N$, it corresponds with a vector in $\mathbb{R}^{2^{n}-1}$. Let $|S|$ be the number of elements of coalition $S$. Saving notation, since the set $N$ is fixed throughout the paper, we denote a game by $v$; also, when no ambiguity arises, we use $i$ to denote $\{i\}$. A game $v$ is additive if, for each $S, T \subseteq N$ such that $T \cap S=\emptyset$, $v(S \cup T)=v(S)+v(T)$.

Let $v$ be a TU game and let $x \in \mathbb{R}^{n}$ be an allocation. Then, $x$ is efficient if $\sum_{i=1}^{n} x_{i}=$ $v(N) ; x$ is individually rational if, for each $i \in N, x_{i} \geq v(i)$. The imputation set is defined by $I(v):=\left\{x \in \mathbb{R}^{n}: \sum_{i \in N} x_{i}=v(N)\right.$ and, for each $\left.i \in N, x_{i} \geq v(i)\right\}$, i.e., the set of all efficient and individually rational allocations.

Let $v \in G^{n}$ and let $i, j \in N$. Then, $i$ and $j$ are symmetric if, for each $S \subseteq N \backslash\{i, j\}$, $v(S \cup i)-v(S)=v(S \cup j)-v(S) ; i$ and $j$ are quasi-symmetric if, for each $S \subseteq N \backslash\{i, j\}$, $v(S \cup i)-(v(S)+v(i))=v(S \cup j)-(v(S)+v(j))$. Now, $v$ is symmetric if, for each pair $i, j \in N, i$ and $j$ are symmetric; $v$ is quasi-symmetric if, for each pair $i, j \in N, i$ and $j$ are quasi-symmetric or, equivalently, a game is quasi-symmetric if the corresponding 
0-normalized game is symmetric. Note that, for a symmetric game, $v(S)$ only depends on the cardinality of $S$.

An allocation rule is a function which, given a game $v$, selects an allocation in $\mathbb{R}^{n}$, i.e.,

$$
\begin{array}{rll}
\varphi: \Omega \subseteq G^{n} & \longrightarrow & \mathbb{R}^{n} \\
v & \longmapsto & \varphi(v) .
\end{array}
$$

Next, we define some properties for allocation rules. Let $v \in G^{n}$ and let $\varphi$ be an allocation rule: $\varphi$ is continuous if the function $\varphi: \Omega \subseteq \mathbb{R}^{2^{n}-1} \rightarrow \mathbb{R}^{n}$ is continuous; $\varphi$ is efficient if it always select efficient allocations; $\varphi$ is translation invariant if, for each two games $v$ and $w$ and each $\alpha=\left(\alpha_{1}, \ldots, \alpha_{n}\right) \in \mathbb{R}^{n}$ such that, for each $S \subseteq N, w(S)=$ $v(S)+\sum_{i \in S} \alpha_{i}$, then $\varphi(w)=\varphi(v)+\alpha$. We say that $\varphi$ satisfies weak symmetry if, for each symmetric game $v$ and each pair $i, j \in N, \varphi_{i}(v)=\varphi_{j}(v) ; \varphi$ satisfies extended weak symmetry if, for each quasi-symmetric game $v$ and each pair $i, j \in N, \varphi_{i}(v)-v(i)=\varphi_{j}(v)-v(j)$. Under extended weak symmetry, if, for each pair $i, j \in N$, their contribution to any coalition differs only in $v(i)-v(j)$, then the difference in the payoffs is also $v(i)-v(j)$. This property, besides being a symmetry property (it implies weak symmetry) has some flavor to translation invariance; roughly speaking, it says that the allocation rule satisfies weak symmetry and, moreover, translation invariance within the class of quasi-symmetric games. The next lemma illustrates this point.

Lemma 1. Translation invariance + weak symmetry $\Rightarrow$ extended weak symmetry.

Proof. Let $\varphi$ be an allocation rule satisfying translation invariance and weak symmetry. Let $v$ be a quasi-symmetric game and $\alpha:=(-v(1), \ldots,-v(n))$. Let $w \in G^{n}$ be the symmetric game defined, for each $S \subseteq N$, by $w(S):=v(S)+\sum_{i \in S} \alpha_{i}$. Hence, by weak symmetry, for each pair $i, j \in N, \varphi_{i}(w)=\varphi_{j}(w)$. Now, by translation invariance, we have $\varphi_{i}(v)+\alpha_{i}=$ $\varphi_{j}(v)+\alpha_{j}$. Since $\alpha_{i}=-v(i)$ and $\alpha_{j}=-v(j)$, the result is proved.

\subsection{The core and its relatives}

We introduce now the notions of core (Gillies, 1953) and strong $\varepsilon$-core (Maschler et al., 1979). An allocation $x \in \mathbb{R}^{n}$ is coalitionally rational if there is no coalition $S \subseteq N$ such that $\sum_{i \in S} x_{i}<v(S)$; analogously, for each $\varepsilon \in \mathbb{R}, x$ is $\varepsilon$-coalitionally rational if there is no coalition $S \subseteq N$ such that $\sum_{i \in S} x_{i}<v(S)-\varepsilon$.

The core of a game $v, C(v)$, is the set of all efficient and coalitionally rational allocations, i.e., $C(v):=\left\{x \in \mathbb{R}^{n}: \sum_{i \in N} x_{i}=v(N)\right.$ and, for each $\left.S \subsetneq N, \sum_{i \in S} x_{i} \geq v(S)\right\}$. Let $B G^{n} \subsetneq G^{n}$ be the class of TU games with nonempty core. Let $\varepsilon \in \mathbb{R}$. The strong $\varepsilon$-core of a game $v, C_{\varepsilon}(v)$ is the set of all efficient and $\varepsilon$-coalitionally rational allocations, i.e., $C_{\varepsilon}(v):=\left\{x \in \mathbb{R}^{n}: \sum_{i \in N} x_{i}=v(N)\right.$ and, for each $\left.S \subsetneq N, \sum_{i \in S} x_{i} \geq v(S)-\varepsilon\right\}$. By definition, if $\varepsilon=0, C_{0}(v) \equiv C(v)$. The least core of $v, \mathcal{L} C(v)$, is the intersection of all 
nonempty strong $\varepsilon$-cores. Equivalently, if $\varepsilon_{0}(v)$ is the smallest $\varepsilon$ such that $C_{\varepsilon}(v) \neq \emptyset$, then $\mathcal{L} C(v)=C_{\varepsilon_{0}(v)}(v) \underline{1}$

Let $v \in G^{n}$ and $\varepsilon \in \mathbb{R}$. In Maschler et al. (1979), the shifted game $v_{\varepsilon}$ is defined by:

$$
v_{\varepsilon}(S):= \begin{cases}v(S)-\varepsilon & \emptyset \neq S \subsetneq N \\ v(S) & S=\emptyset \text { or } S=N .\end{cases}
$$

We present now the core-center, introduced in González-Díaz and Sánchez-Rodríguez (2007). Let $U(A)$ denote the uniform distribution defined over the set $A$ and $E(\mathbb{P})$ the expectation of the probability distribution $\mathbb{P}$. Let $v \in B G^{n}$. The core-center of $v, \mu(v)$, is defined by $\mu(v):=E(U(C(v))$.

\subsection{Some geometric considerations}

We need to introduce some notation and make some considerations regarding the underlying geometry of a TU game. Let $H^{N}:=\left\{x \in \mathbb{R}^{n}: \sum_{i \in N} x_{i}=v(N)\right\}$. All the sets we consider in this paper are contained in $H^{N}$ and hence, we develop all our framework in an $(n-1)$ dimensional space.

A (convex) polytope $P$ is the convex hull of a finite set of points $V=\left\{x^{1}, \ldots, x^{k}\right\}$ in $\mathbb{R}^{n}$ or, equivalently, it is a bounded subset of $\mathbb{R}^{n}$ that can be expressed as the intersection of a finite number of halfspaces. The core of a game, when nonempty, is a polytope (it is the intersection of halfspaces in $\left.H^{N}\right)$. A polytope $P$ is an $m$-polytope if its dimension is $m$, i.e., $m$ is the smallest integer such that $P$ is contained in an $m$-dimensional space. Let $P$ be an $m$-polytope and let $m^{\prime} \geq m$, then, $\operatorname{Vol}_{m^{\prime}}(P)$ denotes the $m^{\prime}$-dimensional volume of $P$. Let $P$ be an $m$-polytope. Then, a set of polytopes $\left\{P_{1}, \ldots, P_{k}\right\}$ defines a dissection of $P$ if (i) $P=\bigcup_{l=1}^{k} P_{l}$ and (ii) for each pair $l, l^{\prime} \in\{1, \ldots, k\}$, with $l \neq l^{\prime}, \operatorname{Vol}_{m}\left(P_{l} \cap P_{l^{\prime}}\right)=0$. We present now a result taken from Maschler et al. (1979).

Lemma 2. Except for the least core, all nonempty strong $\varepsilon$-cores are $(n-1)$-polytopes. The least core is always an m-polytope with $m<n-1$.

If the core of a game in $G^{n}$ is an $(n-1)$-polytope, we say it is full dimensional. Otherwise, it is degenerate. By definition, all the restrictions in the core of a game are as follows: let $S \subsetneq N, R^{S}:=\left\{x \in \mathbb{R}^{n}: \sum_{i \in S} x_{i} \geq v(S)\right\}$. We say that the halfspace $R^{S}$ is a $|S|$-restriction. The 1-restrictions play a special role in this paper; we call them elemental restrictions. A restriction is redundant in the core if removing it does not change the core. Conversely, the restrictions that are not redundant are active.

\footnotetext{
${ }^{1}$ In Maschler et al. (1979) it is shown that $\varepsilon_{0}(v)$ exists and is unique.
} 


\section{The trade-off property}

Since the core-center is the expectation of a distribution of probability, it seems unlikely that it can be characterized without using an axiom with some sort of measurement requirement embedded into it:2

Given $A \subset \mathbb{R}^{k}$, the relative interior of $A, A^{\mathrm{ri}}$, is the euclidean interior of $A$ when considered as a subset of its affine hull 3

Definition 1. Let $\varphi$ be an allocation rule defined on $B G^{n}$. Let $v, w_{1}$ and $w_{2}$ be three games in $B G^{n}$ such that $C(v)=C\left(w_{1}\right) \cup C\left(w_{2}\right)$ and $C\left(w_{1}\right)^{\mathrm{ri}} \cap C\left(w_{2}\right)^{\mathrm{ri}}=\emptyset$. Then, $\varphi$ satisfies trade-off in $B G^{n}$ if there is $\alpha \in[0,1]$ such that

$$
\varphi(v)=\alpha \varphi\left(w_{1}\right)+(1-\alpha) \varphi\left(w_{2}\right)
$$
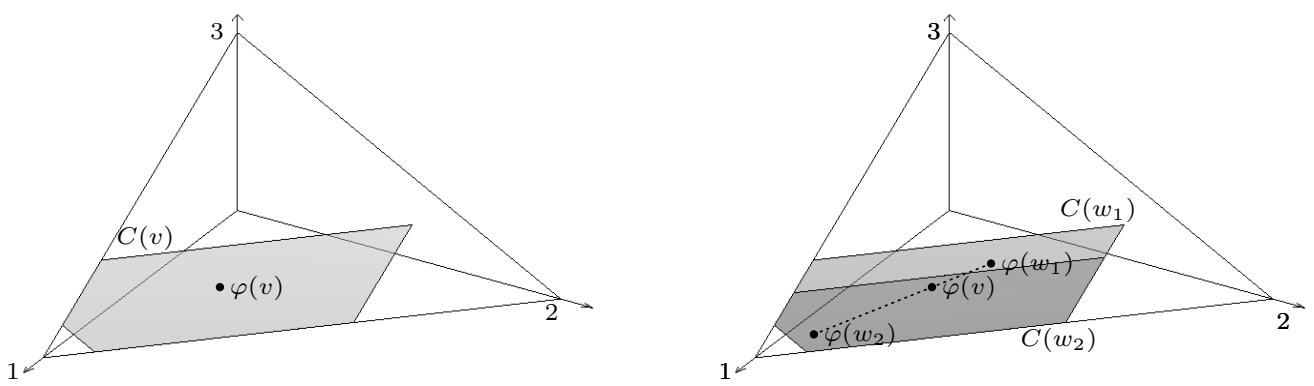

Figure 1: Illustration of the trade-off property

Figure 1 illustrates the above definition: the allocation for $v$ is a trade-off between the allocations for $w_{1}$ and $w_{2}$. The coefficient $\alpha$ measures the importance of games $w_{1}$ and $w_{2}$ with respect to $v$. Whenever there are games $v, w_{1}$ and $w_{2}$ such that $C(v)=$ $C\left(w_{1}\right) \cup C\left(w_{2}\right)$ and $C\left(w_{1}\right)^{\text {ri }} \cap C\left(w_{2}\right)^{\text {ri }}=\emptyset$, there has to be a coalition $T \subsetneq N$ such that, for each $x \in C\left(w_{1}\right)$ and each $y \in C\left(w_{2}\right), \sum_{i \in T} x_{i} \geq \sum_{i \in T} y_{i}$; moreover, under efficiency, $\sum_{i \in N \backslash T} y_{i} \geq \sum_{i \in N \backslash T} x_{i}$. That is, $C\left(w_{1}\right)$ contains the good allocations for $T$ and $C\left(w_{2}\right)$ the good ones for $N \backslash T$. Therefore, if we think of $w_{1}$ as a good game for $T$ and of $w_{2}$ as a good game for $N \backslash T$, then we may see the trade-off property as the result of a give and take between coalitions $T$ and $N \backslash T$.

The last observation above becomes even clearer after the following interpretation of the trade-off property. Let $v \in G^{n}$ and suppose there are $\emptyset \neq T \subsetneq N$ and $k \in \mathbb{R}$ such that

\footnotetext{
${ }^{2}$ There is already some literature in which measure theoretical ideas are used in cooperative game theory. For instance, within two person bargaining problems, there is the equal area solution. Anbarci and Bigelow (1994) interpreted equal area as equal concessions. Later, Calvo and Peters (2000) looked at the underlying dynamic process.

${ }^{3}$ The affine hull of a set is the smallest affine space that contains it.
} 
$v(T) \leq k \leq v(N)-v(N \backslash T)$. We use $k$ to define two games: $v^{+}$, a good game for coalition $T$, and $v^{-}$a good game for coalition $N \backslash T$. Suppose that, because of some change in the situation underlying our TU game, coalition $T$ alone can obtain $k$ instead of $v(T)$. The game $v^{+}$is obtained when introducing this change in $v$, i.e., for each $S \subset N, v^{+}(S):=k$ if $S=T$ and $v^{+}(S):=v(S)$ otherwise. Similarly, we define a game $v^{-}$in which coalition $T$ is worstoff. We do it by letting coalition $N \backslash T$ improve, i.e., for each $S \subset N, v^{-}(S):=v(N)-k$ if $S=N \backslash T$ and $v^{-}(S):=v(S)$ otherwise 4 An extension of the trade-off property outside the class of games with nonempty core would say that a solution $\varphi$ should satisfy that there is $\alpha \in[0,1]$ such that $\varphi(v)=\alpha \varphi\left(v^{+}\right)+(1-\alpha) \varphi\left(v^{-}\right)$. In particular, when the cores of $v, v^{+}$and $v^{-}$are nonempty, they would correspond with those of games $v, w_{1}$ and $w_{2}$ in Figure 1 besides, $C\left(v^{+}\right)$and $C\left(v^{-}\right)$are the two parts of $C(v)$ in which the hyperplane given by $v(T)=k$ cuts $C(v)$.

The trade-off property is already quite demanding and not many classic allocation rules satisfy it. As an example, the equal division solution satisfies the trade-off property. Indeed, any solution $\psi$ such that $\psi(v)=\psi(w)$ whenever $v(N)=w(N)$ satisfies the trade-off property; this would include all the solutions that share $v(N)$ among the players according some fixed proportions, with equal division and "dictatorial" solutions as special cases.

Since the trade-off property imposes no structure on the coefficients associated to different dissections, it is hard to analyze its implications 5 Below we introduce a strengthening of the trade-off property. The idea is to make it more functional by imposing some structure on the coefficients.

Given a game $v \in B G^{n}$, we say that the set of games $\left\{w_{1}, \ldots, w_{k}\right\}$ is a dissection of $v$ if $\left\{C\left(w_{1}\right), \ldots, C\left(w_{k}\right)\right\}$ is a dissection of $C(v)$. Now, the trade-off property can be alternatively defined as follows. Let $\varphi$ be an allocation rule in $B G^{n}$. Let $\left\{w_{1}, w_{2}\right\}$ be a dissection of $v$. Then, $\varphi$ satisfies trade-off if there is $\alpha \in[0,1]$ such that $\varphi(v)=\alpha \varphi\left(w_{1}\right)+$ $(1-\alpha) \varphi\left(w_{2}\right)$. Moreover, suppose now that $\left\{w_{3}, w_{4}\right\}$ is a dissection of $w_{2}$. Then, by the trade-off property, there is $\beta \in[0,1]$ such that $\varphi\left(w_{2}\right)=\beta \varphi\left(w_{3}\right)+(1-\beta) \varphi\left(w_{4}\right)$. Hence, $\left\{w_{1}, w_{3}, w_{4}\right\}$ is a dissection of $v, \varphi(v)=\alpha \varphi\left(w_{1}\right)+(1-\alpha) \beta \varphi\left(w_{3}\right)+(1-\alpha)(1-\beta) \varphi\left(w_{4}\right)$ and $\alpha+(1-\alpha) \beta+(1-\alpha)(1-\beta)=1$. Thus, the trade-off property immediately extends to dissections that can be obtained by continuing with the process we have just described. The next property pushes the above idea a little bit further and also imposes a natural restriction on the coefficients associated with a dissection.

Definition 2. Let $\varphi$ be an allocation rule defined on $B G^{n}$. Let $v \in B G^{n}$ and let

\footnotetext{
${ }^{4}$ Depending on the class of games at hand (such as superadditive or convex), the definitions of $v^{+}$and $v^{-}$ might have to be adjusted if we want to ensure that we remain inside the class at hand. In González-Díaz (2005), these ideas are developed for the class of superadditive games.

${ }^{5}$ In this paper we have defined the trade-off property for the class $B G^{n}$. In an earlier version of this paper, contained in González-Díaz (2005, Chapter 6), a more general approach is taken and a version of the trade-off property that also applies to games with empty core is discussed.
} 
$\left\{w_{1}, \ldots, w_{k}\right\}$ and $\left\{\bar{w}_{1}, \ldots, \bar{w}_{l}\right\}$ be two dissections of $v$. Then, $\varphi$ satisfies strong trade-off in $B G^{n}$ if

i) there are $\alpha_{1}, \ldots, \alpha_{k}$ and $\beta_{1}, \ldots, \beta_{l}$ such that $\varphi(v)=\sum_{i=1}^{k} \alpha_{i} \varphi\left(w_{i}\right)=\sum_{i=1}^{l} \beta_{i} \varphi\left(\bar{w}_{i}\right)$,

ii) if there are $p, q$ such that $C\left(w_{p}\right)$ is a translation of $C\left(\bar{w}_{q}\right)$, then $\alpha_{p}=\beta_{q}$.

The real strengthening with respect to the trade-off property is the one given by ii). We can get an equivalent definition of the strong trade-off property by replacing ii) with ii.a) (translation invariance) if there are $p, q$ such that $w_{p}$ is a translation of $\bar{w}_{q}$, then $\alpha_{p}=\beta_{q}$ and ii.b) (core-dependence) if there are $p, q$ such that $C\left(w_{p}\right)=C\left(\bar{w}_{q}\right)$, then $\alpha_{p}=\beta_{q}$. In particular, ii) implies that, given a game $v$ and a dissection $\left\{w_{1}, \ldots, w_{k}\right\}$, each coefficient $\alpha_{i}$ only depends on $v$ and $C\left(w_{i}\right)$.

\section{Trade-off vs. strong trade-off}

It is easy to see that the solutions discussed above that satisfied the trade-off property also satisfy strong trade-off. Yet, the latter is not helpful to understand the implications of this strengthening of the trade-off property. We briefly elaborate now on these implications. First, we present an example of an allocation rule that satisfies trade-off but not strong trade-off. Then, we argue that the same approach allows to define a huge variety of allocation rules that satisfy trade-off but not strong trade-off. For each $v \in G^{n}$, let $z(v):=(v(N) / n, \ldots, v(N) / n)$, i.e., $z$ coincides with the allocation chosen by applying the equal division principle to game $v$. Given two points $x, y \in \mathbb{R}^{n}$, let $\operatorname{dist}(x, y)$ denote the euclidean distance between $x$ and $y$. Given $v \in B G^{n}$, let $\operatorname{Vol}(v):=\int_{C(v)} 1 d x$, i.e., the $(n-1)$-dimensional volume of $C(v)$. Then, the core-center can be alternatively defined, for each $v \in B G^{n}$, by $\mu(v):=\int_{C(v)} x \frac{1}{\operatorname{Vol}(v)} d x$. Now, for each $v \in B G^{n}$, let $M(v):=\int_{C(v)} \frac{1}{1+\operatorname{dist}(x, z(v))} d x$. Consider now the allocation rule $\gamma$ defined in $B G^{n}$ as follows. For each $v \in B G^{n}$,

$$
\gamma(v):=\int_{C(v)} \frac{x}{1+\operatorname{dist}(x, z(v))} \frac{1}{M(v)} d x .
$$

That is, $\gamma(v)$ selects a point in $C(v)$ in a similar way as the core-center does. The main difference is that the core-center uses a uniform distribution over $C(v)$ and $\gamma$ puts more weight on those points that are close to the equal division allocation, $z(v)$. Hence, although $\gamma(v)$ is different from equal division, in general it selects allocations that are closer to equal division than the ones given by the core-center. The allocation rule $\gamma$ satisfies a good number of standard properties such as efficiency, symmetry, dummy player property and continuity 6 Clearly, since the weight of a core in a dissection of $v$ depends on its relative location with respect to $z(v), \gamma$ does not satisfy strong trade-off] 7 We show now that $\gamma$

\footnotetext{
${ }^{6}$ Continuity would be a consequence of Theorem 3 in González-Díaz and Sánchez-Rodríguez (2007).

${ }^{7}$ The fact that $\gamma$ does not satisfy strong trade-off can be formally shown as an easy corollary of Proposition 2 below.
} 
satisfies trade-off. Let $v, w_{1}$ and $w_{2}$ be three games in $B G^{n}$ such that $C(v)=C\left(w_{1}\right) \cup C\left(w_{2}\right)$ and $C\left(w_{1}\right)^{\mathrm{ri}} \cap C\left(w_{2}\right)^{\mathrm{ri}}=\emptyset$. Note that, since $v(N)=w_{1}(N)=w_{2}(N), z(v)=z\left(w_{1}\right)=z\left(w_{2}\right)$. Using that $C\left(w_{1}\right)^{\mathrm{ri}} \cap C\left(w_{2}\right)^{\mathrm{ri}}=\emptyset$ in the second equality below, we get

$$
\begin{aligned}
\gamma(v) & =\int_{C(v)} \frac{x}{1+\operatorname{dist}(x, z(v))} \frac{1}{M(v)} d x \\
& =\int_{C\left(w_{1}\right)} \frac{x}{1+\operatorname{dist}(x, z(v))} \frac{1}{M(v)} d x+\int_{C\left(w_{2}\right)} \frac{x}{1+\operatorname{dist}(x, z(v))} \frac{1}{M(v)} d x \\
& =\frac{1}{M(v)} \int_{C\left(w_{1}\right)} \frac{x}{1+\operatorname{dist}\left(x, z\left(w_{1}\right)\right)} d x+\frac{1}{M(v)} \int_{C\left(w_{2}\right)} \frac{x}{1+\operatorname{dist}\left(x, z\left(w_{2}\right)\right)} d x \\
& =\frac{M\left(w_{1}\right)}{M(v)} \gamma\left(w_{1}\right)+\frac{M\left(w_{2}\right)}{M(v)} \gamma\left(w_{2}\right) .
\end{aligned}
$$

Therefore, taking $\alpha=\frac{M\left(w_{1}\right)}{M(v)}$ and $1-\alpha=\frac{M\left(w_{2}\right)}{M(v)}$, we have that $\gamma$ satisfies the trade-off property. Note that the function $g$ given by $g(x, v(N)):=\frac{1}{1+\operatorname{dist}(x,(v(N) / n, \ldots, v(N) / n))}$ is crucial in the definition of $\gamma$. Actually, $\gamma(v)$ can be seen simply as $\int_{C(v)} x g(x, v(N)) \frac{1}{\int_{C(v)} g(y, v(N)) d y} d x$. If we replace $g$ by the function with constant value 1, we get the core-center. Therefore, by taking different functions instead of $g$, we can use the above approach to define plenty of allocation rules that satisfy trade-off but not strong trade-off. We could take a different reference point, a reference set, different distances, multiply by the distance instead of dividing by it and even further, we could also use functions that have nothing to do with any kind of distance. Essentially, the only property that any such function, namely $f$, has to meet is that, given $v, w_{1}$ and $w_{2}$ in $B G^{n}$ such that $C(v)=C\left(w_{1}\right) \cup C\left(w_{2}\right)$ and $C\left(w_{1}\right)^{\mathrm{ri}} \cap C\left(w_{2}\right)^{\mathrm{ri}}=\emptyset$, then $\int_{C(v)} f(x, v(N)) d x=\int_{C\left(w_{1}\right)} f(x, v(N)) d x+\int_{C\left(w_{2}\right)} f(x, v(N)) d x$.

As we have already said, the strong trade-off property has some translation invariance flavor. For instance, for the core-center, the weight of all points is equal, regardless of their location. On the other hand, in most of the solutions that might be defined as above, the weight of each point depends on the coordinates of the point itself and translation invariance is therefore lost.

We present now the two characterizations of the core-center. The first one shows that, for three-player convex games, the trade-off property along with a symmetry property suffice to characterize the core-center 8 The second characterization applies to the general class $B G^{n}$, but at the cost of using the strong version of the trade-off property.

\section{A characterization for three-player convex games}

We begin this section by introducing some extra notation and a symmetry property. A game $v \in G^{n}$ is convex if, for each $i \in N$ and each $S$ and $T$ such that $S \subseteq T \subseteq N \backslash\{i\}$, $v(S \cup\{i\})-v(S) \leq v(T \cup\{i\})-v(T)$. Let $C G^{n} \subset G^{n}$ denote the class of $n$-player

\footnotetext{
${ }^{8}$ We are grateful to an anonymous referee for suggesting this characterization.
} 
convex games. Convex games have very special properties. Just to name two of them that bear special connexion with our analysis here, i) convex games have nonempty core, i.e., $C G^{n} \subset B G^{n}$, and ii) given a pair of convex games $v$ and $w$, if $C(v)=C(w)$, then $v=w$, i.e., a convex game is unambiguously characterized by its core.

Let $z \in \mathbb{R}^{n}$ and let $r \in \mathbb{R}$. Then, we define the hyperplane $H(z, r):=\left\{x \in \mathbb{R}^{n}: z x=r\right\}$. Let $v \in G^{n}$ and let $H(z, r)$ be a hyperplane in $\mathbb{R}^{n}$. Let $A \subset \mathbb{R}^{n}$. We say that $H(z, r)$ is a symmetry hyperplane for $A$ if $A$ is symmetric with respect to $H(z, r)$, i.e., for each $x \in H(z, r)$ and each $a \in \mathbb{R}, x+z a \in A$ if and only if $x-z a \in A$. A set $A$ is symmetric if the intersection of the symmetry hyperplanes for $A$ with $A$ is a singleton, which is called the center of symmetry of $A$. Note that when the center of symmetry of a set exists, it coincides with its center of gravity (the expectation of the uniform distribution defined over the set). A game $v$ is core-symmetric if $C(v)$ is a symmetric set.

Definition 3. Let $\varphi$ be an allocation rule defined on $C G^{n}$. Let $v \in C G^{n}$ be a coresymmetric game. Then, $\varphi$ satisfies core-symmetry in $C G^{n}$ if $\varphi(v)$ is the center of symmetry of $C(v)$, i.e., $\varphi(v)=\mu(v)$.

The above axiom says that if the symmetries of the core of a convex game suffice to characterize its center, then this should be the chosen allocation. Note that we do not need to restrict to the class of convex games to define the core-symmetry property. However, we consider that it is a property specially sensible within this class of games. That is, since each convex game is uniquely characterized by its core, a symmetry in the core implies a symmetry in the game, whereas this implication is more diffuse outside the class of convex games 9 Indeed, it will be clear from the exposition below that for 3-player convex games, this is a mild property and many well-known solution concepts such as the Shapley value and the nucleolus satisfy it.

The characterization result we present in this section refers to three-player convex games and hence, we restrict now to this class of games. Before presenting the result, we discuss the implications of core-symmetry within this class and its connexions with the standard symmetry properties. To do so, we first give a full characterization of the cores of three player convex games.

\subsection{Cores of three-player convex games and core-symmetry}

We fully describe now the different possibilities for full dimensional cores of games in $C G^{3}$. First, note that if the core of a game in $C G^{3}$ is not full dimensional it will be either a singleton or a segment, both of them being symmetric sets. For notational convenience, for

\footnotetext{
${ }^{9}$ For instance, when working with convex games, the vectors of marginal contributions correspond with the vertices of the core and hence, symmetries of the core imply symmetries in the vectors of marginal contributions. This is not necessarily so when we work with non-convex games.
} 
each two player coalition $\{i, j\}$, we use $v(i j)$ to denote $v(\{i, j\})$. Let $x^{1}:=(v(N), 0,0), x^{2}:=$ $(0, v(N), 0)$ and $x^{3}=(0,0, v(N))$. Now, the vector of marginal contributions associated with the order $i j k$ of the players is given by $m_{i}^{i j k}:=v(i), m_{j}^{i j k}:=v(i j)-v(i)$ and $m_{k}^{i j k}:=$ $v(N)-v(i j)$. For convex games, the core is the convex hull of the vectors of marginal contributions and all of them are extreme points of the core.

Let $v$ be a 0 -normalized game such that $v(13) \geq v(12) \geq v(23)$. We characterize now the full dimensional cores that can arise for any such game and this suffices to (geometrically) characterize the core of any three-player convex game. 0-normalization does not entail any loss of generality because the core of any game is geometrically analogous to the core of its 0 -normalization, which is obtained by a simple translation. On the other hand, for any game $v$, the appropriate relabeling of the names of the players leads to $v(13) \geq v(12) \geq v(23)$ and any such relabeling does not affect the geometry of the core of the game. First, the core of a general three-player convex games is depicted in Figure 2 Figures 2 and 3 contain all the possible full dimensional core configurations in $C G^{3}$; different configurations arise when some of the extreme points (marginal vectors) of the hexagon in Figure 2 collapse into each other and into the $x^{i}$ points. There are only two types of degenerate cores in $C G^{3}: \mathbf{1} \mathbf{A}$ the core is a singleton and hence symmetric, which can only happen if the game is additive and 2A the core is a segment and hence symmetric, which can only happen if $v(N)=v(13)>0$ and $v(12)=v(23)=0$.

Hence, if a game in $C G^{3}$ has a symmetric core we have to be in one of the following cases: $\mathbf{1 A}, \mathbf{2 A}, \mathbf{3 A}, \mathbf{3 B}, \mathbf{4 A}$ or $\mathbf{6 B}$, i.e., all cores with 1,2 or 3 extreme points are symmetric, those cores with 4 extreme points that are symmetric fall in case $\mathbf{4 A}$, no core with 5 extreme points is symmetric and a core with 6 extreme points is symmetric if and only if it belongs to case $\mathbf{6 B}$. Note that case $\mathbf{4 A}$ is just a parallelogram, not necessarily a rhombus and that case $6 \mathrm{~B}$ needs not be a regular hexagon.

We are now ready to discuss the implications of the core-symmetry property within the class of three-player convex games. First, most allocations would select the center of the core in cases $\mathbf{1 A}$ and $\mathbf{2 A}$. For instance, any allocation rule satisfying translation invariance and efficiency would select the center of the core for games in case $\mathbf{1 A}$. For the case $\mathbf{2 A}$ it would suffice to add the dummy player property and that symmetric players get the same payoff. Now, for full dimensional cores, with the exception of case $\mathbf{4 A}$, all symmetric cores correspond to symmetric games and hence, any allocation rule satisfying symmetry and efficiency would select the center of the core. Hence, if we add translation invariance, we would get that the allocation rule would select the center of the core of any convex game whose core is symmetric (with the exception of $\mathbf{4 A}$ ). Therefore, case $\mathbf{4 A}$ requires some special discussion. When the core of a three-player convex game is a parallelogram, it is not symmetric according to any of the standard symmetry definitions for cooperative games. Yet, if a three-player convex game $v$ with $N=\{i, j, k\}$ is such that $v(i, j)=0, v(i, k)>0$, 


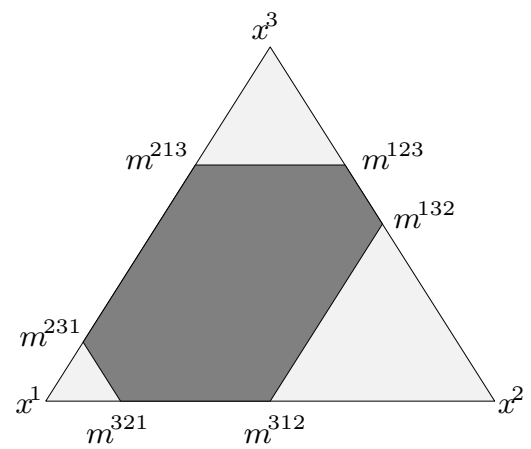

Figure 2: Core of a general game in $C G^{3}$. General case: $6 \mathbf{A}$

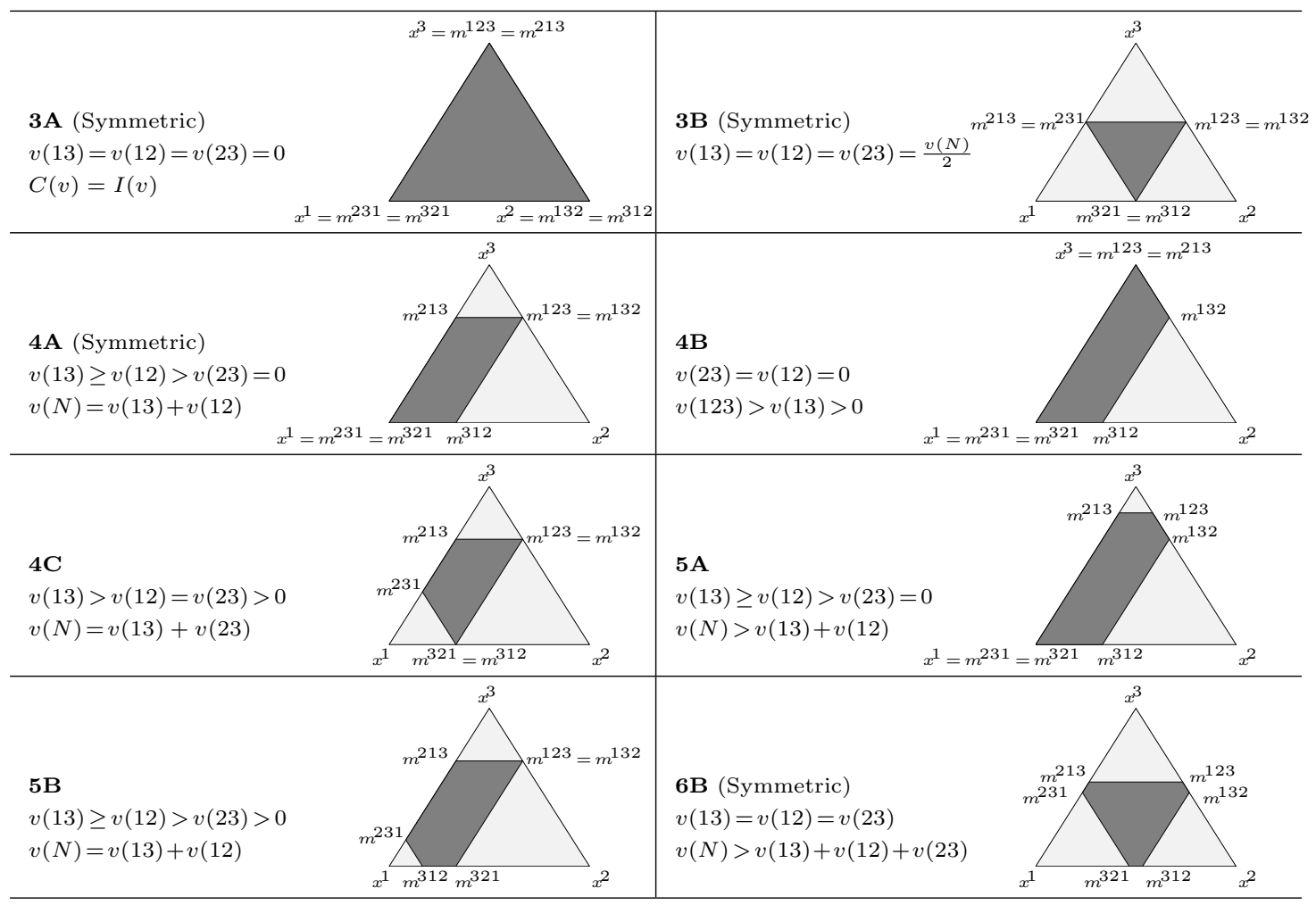

Figure 3: Full dimensional cores of three-player convex games 
$v(j, k)>0$, and $v(N)=v(i, k)+v(j, k)$, it is clear that there is a strong symmetry between players $i$ and $j$. Indeed, it is easy to check that many solutions would select the allocation $x \in \mathbb{R}^{3}$ such that $x_{i}=\frac{v(i, k)}{2}, x_{j}=\frac{v(j, k)}{2}$ and $x_{k}=\frac{v(i, k)}{2}+\frac{v(j, k)}{2}$ in such a game; among them, the Shapley value and the nucleolus. Therefore, the above discussion shows that core-symmetry is not a demanding axiom within $C G^{3}$.

\subsection{The characterization result in $C G^{3}$}

The characterization below shows that core-symmetry and trade-off suffice to characterize the core-center in $C G^{3}$. The independence of the axioms is straightforward. Both Shapley value and nucleolus satisfy core-symmetry and equal division satisfies trade-off.

Theorem 1. Let $\varphi$ be an allocation rule defined on $C G^{3}$ and satisfying core-symmetry and trade-off. Then, for each $v \in C G^{3}, \varphi(v)=\mu(v)$.

Proof. Let $v \in C G^{n}$. We show that, regardless of the number of extreme points of $C(v)$, $\varphi(v)=\mu(v)$. If $C(v)$ has less than 4 extreme points, then it is core-symmetric and, by core-symmetry, $\varphi(v)=\mu(v)$. Suppose now that $C(v)$ has 4 extreme points. In case $\mathbf{4} \mathbf{A}$, $\varphi(v)=\mu(v)$ follows again from core-symmetry. We prove now that if $C(v)$ is such as in case $4 \mathbf{B}$, then $\varphi(v)=\mu(v)$. We assume, without loss of generality, that $v$ is a 0 -normalized game; the proof can be immediately adapted for the general case just by taking the corresponding translations of all the games defined below. Moreover, relabeling the players if needed, assume that $v(13) \geq v(12) \geq v(23)$. Let $k:=v(13)$. Let $v^{+}$be defined by $v^{+}(3):=k$, $v^{+}(23):=k$ and $v^{+}(S):=v(S)$ otherwise. Let $v^{-}$be defined by $v^{-}(12):=v(N)-k$ and $v^{-}(S):=v(S)$ otherwise. Clearly, both $v^{+}$and $v^{-}$are convex games and, moreover, they "cut" $C(v)$ at "height" $k$ and $C(v)=C\left(v^{+}\right) \cup C\left(v^{-}\right)$and $C\left(v^{+}\right)^{\mathrm{ri}} \cap C\left(v^{-}\right)^{\mathrm{ri}}=\emptyset$; the central picture in Figure 4 illustrates the construction. Now, it is easy to see that $C\left(v^{+}\right)$belongs to case $\mathbf{3} \mathbf{A}$ and $C\left(v^{-}\right)$belongs to case $\mathbf{4} \mathbf{A}$. Hence, by core-symmetry, $\varphi\left(v^{+}\right)=\mu\left(v^{+}\right)$ and $\varphi\left(v^{-}\right)=\mu\left(v^{-}\right)$. By the trade-off property, there is $\alpha \in[0,1]$ such that $\varphi(v)=$ $\alpha \varphi\left(v^{+}\right)+(1-\alpha) \varphi\left(v^{-}\right)=\alpha \mu\left(v^{+}\right)+(1-\alpha) \mu\left(v^{-}\right)$. That is, $\varphi(v)$ lies in the segment joining $\mu\left(v^{+}\right)$and $\mu\left(v^{-}\right)$. Clearly, by construction, the center of $\mu(v)$ belongs to this segment. Similarly, let $w^{+}$be defined by $w^{+}(1):=k, w^{+}(12):=k$ and $w^{+}(S):=v(S)$ otherwise. Let $w^{-}$be defined by $w^{-}(23):=v(N)-k$ and $w^{-}(S):=v(S)$ otherwise. Again, $w^{+}$and $w^{-}$are convex games and $C(v)=C\left(w^{+}\right) \cup C\left(w^{-}\right)$and $C\left(w^{+}\right)^{\mathrm{ri}} \cap C\left(w^{-}\right)^{\mathrm{ri}}=\emptyset$ (see the right picture in Figure (4). Now, $C\left(w^{+}\right)$belongs to case $\mathbf{3 A}$ and $C\left(w^{-}\right)$belongs to case $4 \mathbf{A}$. Hence, by core-symmetry, $\varphi\left(w^{+}\right)=\mu\left(w^{+}\right)$and $\varphi\left(w^{-}\right)=\mu\left(w^{-}\right)$. By the trade-off property, there is $\beta \in[0,1]$ such that $\varphi(v)=\beta \mu\left(w^{+}\right)+(1-\beta) \mu\left(w^{-}\right)$. That is, $\varphi(v)$ lies in the segment joining $\mu\left(w^{+}\right)$and $\mu\left(w^{-}\right)$. Again, the construction ensures that $\mu(v)$ belongs to this segment as well. Moreover, it is also easy to see that the way the two "cuts" have been defined ensures that the two segments are not parallel to each other, so they intersect at most in one point. 
Hence, since $\mu(v)$ belongs to both segments, we have that $\varphi(v)=\mu(v)$. The proof for case $\mathbf{4 C}$ follows from an analogous construction.
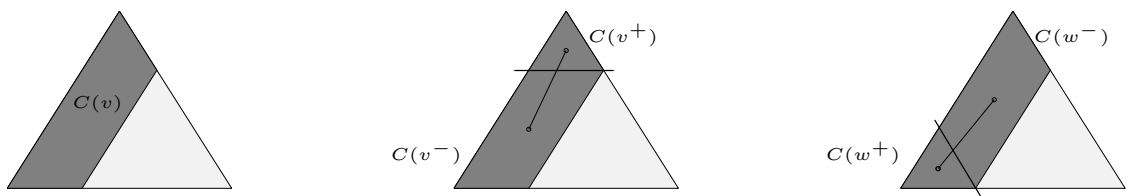

Figure 4: The case 4B

The proof is now easily extended to cores with 5 extreme points. Clearly, for any game whose core is such as in case $\mathbf{5 A}$ or $\mathbf{5 B}$, there are at least two different ways in which we can "cut" its core and divide it in two cores with, at most, 4 extreme points. Since $\varphi$ has already been characterized for any game whose core has at most 4 points, we can show that $\varphi(v)=\mu(v)$ following the same argument as with case $4 \mathrm{~B}$ above. Finally, once that any game whose core has 5 extreme points has been characterized, if we are given a game with 6 extreme points, there are at least two different ways in which we can "cut" its core and divide it in two cores with, at most, 5 extreme points and the same argument applies again.

The natural question now is how to generalize the above result beyond three-player games (and maybe beyond convex games). There are several difficulties in this task. Essentially, it would require a characterization of the symmetric cores that arise in any convex game, which is not an easy task. Moreover, we argued above that core-symmetry is a mild requirement in $C G^{3}$, but it is not clear whether this would also be the case in general in $C G^{n}$ and this issue should also be addressed. Another approach would be to replace coresymmetry with standard axioms such as translation invariance, symmetry and efficiency and this is what we do in the following characterization. The problem with this approach is that there are cases of games with some structural symmetries such as the parallelogram in case $\mathbf{4 A}$ above (and others when there are more than three players) in which the above axioms do not suffice to characterize the allocation to select there. Because of this, we could only succeed in our characterization after strengthening trade-off to strong trade-off.

\section{A characterization for $n$-player games with nonempty core}

The strong trade-off property is the essential element of this second characterization of the core-center. 
Theorem 2. Let $\varphi$ be an allocation rule defined on $B G^{n}$ and satisfying

\section{T1) Efficiency}

T2) Continuity

T3) Extended weak symmetry

\section{T4) Strong trade-off}

Then, for each $v \in B G^{n}, \varphi(v)=\mu(v)$.

The core-center is efficient because it lies in the core. The issue of continuity, despite not being straightforward, has already been positively addressed in González-Díaz and SánchezRodríguez (2007). Moreover, it is trivial that the core-center satisfies weak symmetry and translation invariance and hence, by Lemma 1, also extended weak symmetry. The strong trade-off property is an immediate consequence of the properties of the center of gravity, the coefficients being proportional to the cores of the games in the dissection. Remarkably, no axiom requires that the allocation rule selects core allocations; we discuss how this property is implied by the others in the remark at the end of Section 5.2, which we devote to prove Theorem 2 .

The strong trade-off property is seemingly stronger than the trade-off property. Roughly speaking, the coefficients are required to be translation invariant and equal for equally shaped cores with the same size. Hence, one might conjecture that the core-center is the unique allocation rule satisfying i) efficiency, ii) continuity, iii) weak symmetry, iv) translation invariance, v) core dependence 10 and vi) trade-off 11 Although we acknowledge that this alternative characterization would be more natural because the axioms of translation invariance and core dependence are explicit (instead of embedded in the strong trade-off property), we have not succeeded in proving it. The main problem we have faced is that, given an allocation rule satisfying the above six axioms, we have not been able to use the translation invariance and core dependence of the allocation rule to pin down the coefficients given by the trade-off property. Essentially, since the trade-off property imposes no structure at all on the coefficients, we have not been able to do anything similar to what we do in Section 5.2 to get Proposition 2, there we show that if the coefficients themselves satisfy translation invariance and core dependence, then they have to be proportional to the volumes. Indeed, note that the allocation rule $\gamma$ defined at the end of Section 3 verifies all the 5 properties above with the exception of translation invariance (actually, it does even satisfy not only weak symmetry, but symmetry).

We acknowledge that a characterization that relies on the strong trade-off property instead of doing it just on the trade-off property is less appealing. Nonetheless, we hope

\footnotetext{
${ }^{10}$ An allocation rule satisfies core dependence if, whenever $C(v)=C(w)$, then $\varphi(v)=\varphi(w)$.

${ }^{11}$ Or, maybe, efficiency needs to be replaced by core selection and weak symmetry by anonymity.
} 
that our results may shed some light for a future characterization in which no further structure needs to be imposed on the trade-off property.

\subsection{Tightness of the characterization}

Next, we prove that the properties in Theorem 2 are tight. In order to do this we need the next lemma.

Lemma 3. Let $v \in B G^{n}$ be a quasi-symmetric game. Then, $C(v)$ either is a point or is full dimensional.

Proof. Recall that the core of a quasi-symmetric game can be transformed into the one of a symmetric game just using a translation. To prove this lemma it suffices to show that the result is true for symmetric games. Hence, let $v$ be a symmetric game, and assume that it has a degenerate core. Hence, there is $S \subsetneq N$ such that, for each $x \in C(v), \sum_{i \in S} x_{i}=v(S)$. Let $k:=v(S)$. Now, by symmetry, for each $x \in C(v)$ and each $|S|$-player coalition $S^{\prime}$, we have $\sum_{i \in S^{\prime}} x_{i}=k$. Clearly, this only happens if, for each $i \in N, x_{i}=\frac{k}{|S|}$. Now, by efficiency, $k=|S| v(N) / n$. Hence $C(v)=\{x\}$, where, for each $i \in N, x_{i}=v(N) / n$.

Proposition 1. None of the properties used in Theorem 2 to characterize the core-center is redundant.

Proof. Next, we show that if we remove one of these properties there are allocation rules different from the core-center satisfying the remaining ones.

Remove strong trade-off: Both Shapley value and nucleolus satisfy efficiency, extended weak symmetry, and continuity.

Remove efficiency: Take $k \neq 0$. The allocation rule $\varphi(v)=\mu(v)+(k, \ldots, k)$ satisfies strong trade-off, extended weak symmetry, and continuity.

Remove extended weak symmetry: The allocation rule $\varphi(v)=(v(N), 0, \ldots, 0)$ satisfies strong trade-off, efficiency, and continuity.

Remove continuity: We need to distinguish different cases:

The core is a single point: $\varphi$ selects the point (the core-center).

The core is degenerate but not a single point: In this case the allocation $\varphi$ selects the point $(v(N), 0, \ldots, 0)$.

The core is not degenerate: $\varphi$ selects the core-center.

This allocation rule satisfies strong trade-off, efficiency and extended weak symmetry. The strong trade-off property follows from assigning to each game in a dissection a coefficient that is proportional to the volume of its core. Efficiency is straightforward. 
It also satisfies extended weak symmetry: in the non-degenerate case it coincides with the core-center so extended weak symmetry is met; in the degenerate case, as a consequence of Lemma 3 there are no quasi-symmetric games with degenerate core with more than one point and in the latter cases, the point is the selected allocation so extended weak symmetry is satisfied.

\subsection{Proof of the characterization}

This section is entirely devoted to prove Theorem 2 First, we present a preliminary but crucial result whose idea is similar to that of a classical result in measure theory, namely, "If $m$ is the Lebesgue measure, and $\eta$ is a positive translation invariant Borel measure on $\mathbb{R}^{k}$ such that $\eta(K)<\infty$ for every compact set $K$, then there is a constant $c$ such that $\eta(E)=c m(E)$ for all Borel sets $E \subset \mathbb{R}^{k}$ " (Rudin, 1966). Recall that, under the strong trade-off property, given a game $v$ and a dissection $\left\{w_{1}, \ldots, w_{k}\right\}$, each coefficient $\alpha_{i}$ only depends on $v$ and $C\left(w_{i}\right)$; hence, we denote it by $\alpha_{v}\left(w_{i}\right)$.

Proposition 2. Let $\varphi$ be an allocation rule satisfying the strong trade-off property. Let $v$ and $v^{\prime}$ be two games in $B G^{n}$ such that $v^{\prime}$ belongs to some dissection of $v$. If $C(v)$ is an $m$-polytope, then $\alpha_{v}\left(v^{\prime}\right)=\frac{\operatorname{Vol}_{m}\left(C\left(v^{\prime}\right)\right)}{\operatorname{Vol}_{m}(C(v))}$.

Proof. Let $v \in B G^{n}$ and let $C(v)$ be an $m$-polytope. For each $v^{\prime} \in B G^{n}$ belonging to some dissection of $v$, let $w\left(v^{\prime}\right):=\alpha_{v}\left(v^{\prime}\right) \operatorname{Vol}_{m}(C(v))$. We have to prove that $w(\cdot)=\operatorname{Vol}_{m}(C(\cdot))$. Hence, in the rest of the proof, we restrict attention to games that belong to some dissection of $v$. First, we show that there is $\varepsilon>0$ such that if $\operatorname{Vol}_{m}\left(C\left(v^{\prime}\right)\right)<\varepsilon$, then $w\left(v^{\prime}\right)=$ $\operatorname{Vol}_{m}\left(C\left(v^{\prime}\right)\right)$. Suppose, on the contrary, that, for each $\varepsilon>0$, there is $v_{\varepsilon}$ such that $w\left(v_{\varepsilon}\right) \neq$ $\operatorname{Vol}_{m}\left(C\left(v_{\varepsilon}\right)\right)<\varepsilon$. We distinguish two cases:

Case 1: For each $\varepsilon>0$, there is $v_{\varepsilon}$ such that $\operatorname{Vol}_{m}\left(C\left(v_{\varepsilon}\right)\right)<\varepsilon$ and $w\left(v_{\varepsilon}\right)>\operatorname{Vol}_{m}\left(C\left(v_{\varepsilon}\right)\right)$. Let $\varepsilon>0$ and $v_{\varepsilon}$ be as above. Let $\delta=w\left(v_{\varepsilon}\right)-\operatorname{Vol}_{m}\left(C\left(v_{\varepsilon}\right)\right)$. Let $\left\{v_{\varepsilon_{k}}\right\}_{k \in \mathbb{N}}$ be such that $\left\{\varepsilon_{k}\right\} \rightarrow 0$ and, for each $k \in \mathbb{N}, \varepsilon_{k}>0$ and $w\left(v_{\varepsilon_{k}}\right)>\operatorname{Vol}_{m}\left(C\left(v_{\varepsilon_{k}}\right)\right)$ and $\operatorname{Vol}_{m}\left(C\left(v_{\varepsilon_{k}}\right)\right)<\varepsilon_{k}$. Now, let $\mathcal{G} \subset B G^{n}$ be a dissection of $v$ such that $\mathcal{G}:=v_{\varepsilon} \cup \mathcal{G}_{1} \cup \mathcal{G}_{2}$, where $\mathcal{G}_{1}$ satisfies i) for each $\hat{v} \in \mathcal{G}_{1}$, there is $k \in \mathbb{N}$ such that $C(\hat{v})$ is a translation of $C\left(v_{\varepsilon_{k}}\right)$ and ii) $\sum_{\hat{v} \in \mathcal{G}_{1}} \operatorname{Vol}_{m}(C(\hat{v}))>$ $\operatorname{Vol}_{m}(C(v))-\operatorname{Vol}_{m}\left(C\left(v_{\varepsilon}\right)\right)-\delta$. Then, since $\varphi$ satisfies strong trade-off, for each $\hat{v} \in \mathcal{G}_{1}$ there is $k \in \mathbb{N}$ such that, $w(\hat{v})=w\left(v_{\varepsilon_{k}}\right)>\operatorname{Vol}_{m}\left(C\left(v_{\varepsilon_{k}}\right)\right)=\operatorname{Vol}_{m}(C(\hat{v}))$. Hence, $\sum_{\hat{v} \in \mathcal{G}} w(\hat{v}) \geq$ $w\left(v_{\varepsilon}\right)+\sum_{\hat{v} \in \mathcal{G}_{1}} w(\hat{v})>\operatorname{Vol}_{m}\left(C\left(v_{\varepsilon}\right)\right)+\delta+\operatorname{Vol}_{m}(C(v))-\operatorname{Vol}_{m}\left(C\left(v_{\varepsilon}\right)\right)-\delta=\operatorname{Vol}_{m}(C(v))$. Therefore, $\sum_{\hat{v} \in \mathcal{G}} \alpha_{v}(\hat{v})>1$ and we have a contradiction.

Case 2: For each $\varepsilon>0$, there is $v_{\varepsilon}$ such that $\operatorname{Vol}_{m}\left(C\left(v_{\varepsilon}\right)\right)<\varepsilon$ and $w\left(v_{\varepsilon}\right)<\operatorname{Vol}_{m}\left(C\left(v_{\varepsilon}\right)\right)$. We claim that Case 2 implies Case 1. Let $\left\{v_{\varepsilon_{k}}\right\}_{k \in \mathbb{N}}$ be such that $\left\{\varepsilon_{k}\right\} \rightarrow 0$ and, for each $k \in \mathbb{N}, \varepsilon_{k}>0$ and $w\left(v_{\varepsilon_{k}}\right)<\operatorname{Vol}_{m}\left(C\left(v_{\varepsilon_{k}}\right)\right)<\varepsilon_{k}$. For each $\delta>0$, let $\mathcal{G}_{\delta} \in B G^{n}$ be a dissection of $v, \mathcal{G}_{\delta}:=\mathcal{G}_{1} \cup \mathcal{G}_{2}$, where $\mathcal{G}_{1}$ satisfies i) for each $\hat{v} \in \mathcal{G}_{1}$, there is $k \in \mathbb{N}$ such that $C(\hat{v})$ is a translation of $C\left(v_{\varepsilon_{k}}\right)$ and ii) $\sum_{\hat{v} \in \mathcal{G}_{1}} \operatorname{Vol}_{m}(C(\hat{v}))>\operatorname{Vol}_{m}(C(v))-\delta$. Then, 
since $\varphi$ satisfies strong trade-off, for each $\hat{v} \in \mathcal{G}_{1}, w(\hat{v})=w\left(v_{\varepsilon_{k}}\right)$. Since $\sum_{\hat{v} \in \mathcal{G}} \alpha_{v}(\hat{v})=1$, $\sum_{\hat{v} \in \mathcal{G}} w(\hat{v})=\operatorname{Vol}_{m}(C(v))$. Hence, there is $v_{\delta} \in \mathcal{G}_{2}$ such that $\operatorname{Vol}_{m}\left(C\left(v_{\delta}\right)\right)<\delta$ and $w\left(v_{\delta}\right)>$ $\operatorname{Vol}_{m}\left(C\left(v_{\delta}\right)\right)$. Therefore, we are in Case 1.

Hence, we have shown that there is $\varepsilon>0$ such that if $\operatorname{Vol}_{m}\left(C\left(v^{\prime}\right)\right)<\varepsilon$, then $w\left(v^{\prime}\right)=$ $\operatorname{Vol}_{m}\left(C\left(v^{\prime}\right)\right)$. Now, for each game $v^{\prime} \in B G^{n}$ belonging to a dissection of $v$, we can dissect $v^{\prime}$ in such a way that the cores of the games of the dissection cover $C\left(v^{\prime}\right)$ and their volumes are less than $\varepsilon$. Hence, $w\left(v^{\prime}\right)=\operatorname{Vol}_{m}\left(C\left(v^{\prime}\right)\right)$.

Corollary 1. Let $\varphi$ be an allocation rule satisfying the strong trade-off property. Let $v \in$ $B G^{n}$ and $\left\{v_{1}, \ldots, v_{r}\right\} \subset B G^{n}$ dissection of $v$. If $C(v)$ is an m-polytope, then

$$
\varphi(v)=\sum_{i=1}^{r} \alpha_{v}\left(v_{i}\right) \varphi\left(v_{i}\right), \quad \text { where } \alpha_{v}\left(v_{i}\right)=\frac{\operatorname{Vol}_{m}\left(C\left(v_{i}\right)\right)}{\operatorname{Vol}_{m}(C(v))} .
$$

Proof. Immediate from Proposition 2

The above two results imply that, for any allocation rule satisfying strong trade-off, the weights of the games in a dissection contained in $B G^{n}$ have to be proportional to the volumes of their cores. This is a crucial result to characterize the core-center.

The outline of the proof of Theorem 2 is as follows.

Step 1: We characterize the core-center when the core is simple enough. (Section 5.2.1).

Step 2: We show that the four properties T1-T4 characterize the core-center for the class of games with full dimensional core (Section 5.2.2).

Step 3: We show that the core of a game in $B G^{n}$ can be approximated by full dimensional cores. Hence, the previous results along with the continuity property lead to the proof of Theorem 2 (Section 5.2.3).

In the first two steps we study full dimensional cores. We only deal with the degenerate case, when the core coincides with the least core, in the last step.

\subsubsection{An elemental core}

Let $v \in B G^{n}$, and let $A$ denote the set of active restrictions in $C(v)$. We say $C(v)$ is elemental if it is full dimensional and $A=\left\{x_{i} \geq v(i): i \in N\right\}=\{$ elemental restrictions $\}$. By definition, if $C(v)$ is an elemental core, then $C(v)=I(v)$.

Lemma 4. Let $v \in B G^{n}$ be such that $C(v)$ is elemental. Then, $C(v)$ is a simplex and its center of gravity is the allocation $x$ such that, for each $i \in N, x_{i}=\frac{v(N)-\sum_{j \in N} v(j)}{n}+v(i)$. 
Proof. Since $C(v)$ is elemental, $C(v)=I(v)$ and $I(v)$ is a simplex with vertices $u^{1}, \ldots, u^{n}$, where, for each $i \in N$ and each $j \neq i, u_{j}^{i}=v(j)$ and $u_{i}^{i}=v(N)-\sum_{j \neq i} v(j)$. Hence, to obtain the result, we only need to calculate the center of gravity of the simplex, i.e., the average of the vertices.

Proposition 3. Let $v \in B G^{n}$ be such that $C(v)$ is elemental. Let $\varphi$ be an allocation rule satisfying efficiency, extended weak symmetry, continuity and strong trade-off. Then, $\varphi(v)=\mu(v)$.

Proof. Since $C(v)$ is elemental, it coincides with the simplex $I(v)$, which is either a point or an $(n-1)$-polytope. Suppose that $I(v)$ is an $(n-1)$-polytope. Let $w \in B G^{n}$ be defined by $w(N):=v(N)$ and, for each $S \subsetneq N, w(S):=\sum_{i \in S} v(i)$. Clearly, $C(w)=I(w)=I(v)=$ $C(v)$. We show that $\varphi(w)=\varphi(v)$. Fix $i \in N$ and let $\bar{w} \in B G^{n}$ be defined, for each $S \subset N$, $S \neq N \backslash\{i\}$, by $\bar{w}(S):=w(S)$ and $\bar{w}(N \backslash\{i\}):=v(N)-v(i)$. So defined, $C(\bar{w})$ is a face of $C(w)$ (it lies in the hyperplane $x_{i}=v(i)$ ). Therefore, $\{C(w), C(\bar{w})\}$ is a dissection of $C(v)$. By the trade-off property, there is $\alpha \in[0,1]$ such that $\varphi(v)=\alpha \varphi(w)+(1-\alpha) \varphi(\bar{w})$. Yet, since $C(v)$ is an $(n-1)$-polytope and $C(\bar{w})$ is an $(n-2)$-polytope, by Corollary $1-\alpha=0$ and hence, $\varphi(v)=\varphi(w)$.

We show now that, $\varphi(w)=\mu(w)$ and, since $C(w)=C(v)$, we have that $\varphi(v)=\varphi(w)=$ $\mu(w)=\mu(v)$. Note that $w$ is quasi symmetric. Hence, by extended weak symmetry, for each pair $i, j \in N, \varphi_{i}(w)-w(i)=\varphi_{j}(w)-w(j)$. Hence, there is $k \in \mathbb{R}$ such that, for each $i \in N, \varphi_{i}(w)=k+w(i)$. The latter comment, along with the efficiency property, implies that, for each $i \in N, \varphi_{i}(w)=\frac{w(N)-\sum_{j \in N} w(j)}{n}+w(i)$. By Lemma 4 $\varphi(w)$ is the center of gravity of $C(w)$, i.e., the core-center. Hence, $\varphi(w)=\mu(w)$.

Now, if $I(v)$ is a point, let $\left\{v^{k}\right\}_{k \in \mathbb{N}}$ be the sequence of games such that, for each $k \in \mathbb{N}$, $v^{k}(N)=v(N)+\frac{1}{k}$ and, for each $S \subsetneq N, v^{k}(S)=v(S)$. Then, $\left\{v^{k}\right\}_{k \in \mathbb{N}}$ converges to $v$. Since for each $k \in \mathbb{N}, I\left(v^{k}\right)$ is an $(n-1)$-polytope, $\varphi\left(v^{k}\right)=\mu\left(v^{k}\right)$. Hence, since $\mu\left(v^{k}\right)$ converges to $I(v)$, the result follows from the continuity property.

Remark. Note that, if we restrict to the class of superadditive games with nonempty core 12 then we can get Proposition 3 without needing strong trade-off and continuity (any superadditive game whose core coincides with the set of imputations is quasi-symmetric). That is, in that class of games, any allocation rule satisfying efficiency and extended weak symmetry selects the core-center for games with an elemental core.

\subsubsection{The core is full dimensional}

The role of the games with an elemental core is now similar to that of the unanimity games in the characterization of the Shapley value using additivity. Given a game in $B G^{n}$, we can

\footnotetext{
${ }^{12} \mathrm{~A}$ game $v$ is superadditive if, for each $S, T \subseteq N$ such that $T \cap S=\emptyset, v(S \cup T) \geq v(S)+v(T)$.
} 
find dissections of its core mainly composed by elemental cores. Taking to zero the volume of the cores of a dissection that are not elemental and combining Proposition 3 with the continuity and the strong trade-off properties we get the desired result for any game with a non-degenerate core.

Proposition 4. Let $v \in B G^{n}$ be such that $C(v)$ is full dimensional. Let $\varphi$ be an allocation rule satisfying the four properties T1-T4. Then, $\varphi(v)=\mu(v)$.

Proof. Let $\varphi$ be an allocation rule satisfying the properties T1-T4. Let $v \in B G^{n}$ be a game with a full dimensional core. In order to save notation, we denote the cores of games $v$ and $v^{\prime}$ by $C$ and $C^{\prime}$, respectively; moreover, $\operatorname{Vol}(P)$ denotes the $(n-1)$ dimensional volume of polytope $P$. Given a dissection $\mathcal{G}$ of $v$, let $\mathcal{E} \mathcal{G}$ denote the games in $\mathcal{G}$ whose cores are elemental. We can find a sequence $\left\{\mathcal{G}^{t}\right\}_{t \in \mathbb{N}}$ of dissections of $v$ such that i) $\lim _{t \rightarrow \infty} \sum_{v^{\prime} \in \mathcal{G}^{t} \backslash \mathcal{E} \mathcal{G}^{t}} \operatorname{Vol}\left(C\left(v^{\prime}\right)\right)=0$ and ii) there is $m \in \mathbb{R}$ such that, for each $t \in \mathbb{N}$, each $w \in \mathcal{G}^{t}$ and each $S \subseteq N$, we have that $m \leq w(S) \leq v(N) 13$ Now, by the strong trade-off property of $\varphi$ and Corollary 1 .

$$
\varphi(v)=\sum_{v^{\prime} \in \mathcal{G}^{t}} \frac{\operatorname{Vol}\left(C^{\prime}\right)}{\operatorname{Vol}(C)} \varphi\left(v^{\prime}\right)=\frac{1}{\operatorname{Vol}(C)}\left(\sum_{v^{\prime} \in \mathcal{E} \mathcal{G}^{t}} \operatorname{Vol}\left(C^{\prime}\right) \varphi\left(v^{\prime}\right)+\sum_{v^{\prime} \in \mathcal{G}^{t} \backslash \mathcal{E} \mathcal{G}^{t}} \operatorname{Vol}\left(C^{\prime}\right) \varphi\left(v^{\prime}\right)\right) .
$$

By Proposition 3 we have already characterized $\varphi$ for the games in $\mathcal{E G}^{t}$. Moreover, since $\varphi$ is continuous, it is uniformly continuous in the set $B:=\left\{w \in G^{n}\right.$ : for each $S \subseteq N, m \leq$ $w(S) \leq v(N)\}$. Hence, $\varphi$ is bounded in $B$. Since, by construction, all the games in the $\mathcal{G}^{t}$ dissections belong to $B$, we have $\lim _{t \rightarrow \infty} \sum_{v^{\prime} \in \mathcal{G}^{t} \backslash \mathcal{E} \mathcal{G}^{t}} \operatorname{Vol}\left(C^{\prime}\right) \varphi\left(v^{\prime}\right)=0$. Then,

$$
\begin{aligned}
\varphi(v) & =\lim _{t \rightarrow \infty} \sum_{v^{\prime} \in \mathcal{G}^{t}} \frac{\operatorname{Vol}\left(C^{\prime}\right)}{\operatorname{Vol}(C)} \varphi\left(v^{\prime}\right) \\
& =\frac{1}{\operatorname{Vol}(C)}\left(\lim _{t \rightarrow \infty} \sum_{v^{\prime} \in \mathcal{E} \mathcal{G}^{t}} \operatorname{Vol}\left(C^{\prime}\right) \varphi\left(v^{\prime}\right)+\lim _{t \rightarrow \infty} \sum_{v^{\prime} \in \mathcal{G}^{t} \backslash \mathcal{E} \mathcal{G}^{t}} \operatorname{Vol}\left(C^{\prime}\right) \varphi\left(v^{\prime}\right)\right) \\
& =\lim _{t \rightarrow \infty} \frac{1}{\operatorname{Vol}(C)} \sum_{v^{\prime} \in \mathcal{E} \mathcal{G}^{t}} \operatorname{Vol}\left(C^{\prime}\right) \varphi\left(v^{\prime}\right) \\
& \stackrel{\text { Prop } 3}{=} \lim _{t \rightarrow \infty} \frac{1}{\operatorname{Vol}(C)} \sum_{v^{\prime} \in \mathcal{E} \mathcal{G}^{t}} \operatorname{Vol}\left(C^{\prime}\right) \mu\left(v^{\prime}\right) \\
& =\mu(v) .
\end{aligned}
$$

\subsubsection{The core is not full dimensional}

Proposition 5. Let $v \in B G^{n}$ be such that $C(v)$ is not full dimensional. Let $\varphi$ be an allocation rule satisfying the properties T1-T4. Then, $\varphi(v)=\mu(v)$.

\footnotetext{
${ }^{13}$ Refer to González-Díaz (2005, Chapter 6) for a formal proof of the above claim.
} 
Proof. By Lemma 2, $C(v)$ is the least core of $v$. Let $\left\{v_{1 / t}\right\}_{t \in \mathbb{N}}$ be a sequence of shifted games. Now, $\lim _{t \rightarrow \infty} v_{1 / t}=v$. The core of $v_{1 / t}$ coincides with the $\frac{1}{t}$-core of $v$. By Lemma 2 all these $\frac{1}{t}$-cores are full dimensional and hence,

$$
\varphi(v) \stackrel{\text { cont }}{=} \lim _{t \rightarrow \infty} \varphi\left(v_{1 / t}\right) \stackrel{\text { Prop } ⿴ 囗 十}{=} \lim _{t \rightarrow \infty} \mu\left(v_{1 / t}\right) \stackrel{\text { cont }}{=} \mu(v) .
$$

Proof of Theorem 2. The assertion of the theorem follows from Propositions 3, 4, and 5.

Remark. The reasons why core-selection is implied by the other axioms are more transparent now. Proposition 3 implies that, for games with an elemental core, any allocation rule satisfying extended weak symmetry, efficiency and strong trade-off selects the center of the core. Then, the fact that any core can be approximated by elemental cores, along with the trade-off and continuity properties, implies the core selection property for general games with nonempty core.

\section{References}

Anbarci, N. And J. Bigelow (1994): "The Area Monotonic Solution to the Cooperative Bargaining Problem," Mathematical Social Sciences, 28, 133-142.

Calvo, E. And H. Peters (2000): "Dynamics and Axiomatics of the Equal Area Bargaining Solution," International Journal of Game Theory, 29, 81-92.

Gillies, D. B. (1953): "Some Theorems on n-Person Games," Ph.D. thesis, Princeton.

GonzÁlez-DíAz, J. (2005): "Essays on Competition and Cooperation in Game Theoretical Models," Ph.D. thesis, Universidade de Santiago de Compostela.

GonzÁlez-Díaz, J. And E. SÁnchez-Rodríguez (2007): "A natural selection from the core of a TU game: the core-center," International Journal of Game Theory, 36, 27-46, DOI 10.1007/s00182-007-0074-5.

Maschler, M., B. Peleg, and L. S. Shapley (1979): "Geometric Properties of the Kernel, Nucleolus, and Related Solution Concepts," Mathematics of Operations Research, 4, 303-338.

Rudin, W. (1966): Real and Complex Analysis, McGraw-Hill.

Shapley, L. S. (1953): "A Value for $n$-Person Games," in Contributions to the theory of games II, ed. by H. Kuhn and A. Tucker, Princeton: Princeton University Press, vol. 28 of Annals of Mathematics Studies. 